\title{
Subject Index to Volume 20
}

Abstracts, European Society for Pediatric Research, 1034

Acetate, metabolism, perirenal adipose tissue, fetal and neonatal calf, 542

Acetylcholine, gallbladder contractility and, development, guinea pig, 214

$\mathrm{N}$-Acetyl-neuraminic acid, distribution and metabolic fate, infantile sialic acid storage disease, 773

Acetylsalicylic acid, fatty acid hydroxylation and, rat liver, 1221

Acidemia

isovaleric

isovaleryl-CoA dehydrogenase activity, tritium release assay, 59

prenatal diagnosis, 222

metabolic, effect on cardiac output and regional blood flow, newborn lamb, 756

propionic, pancytopenia in, hematologic evaluation, neonate, 783

Acidosis

effect on intestinal blood flow and oxygen uptake, newborn piglet, 929

hypercapnic, effect on renal function, newborn rabbit, 798

lactic, pyruvate carboxylase deficiency, infant, 274

metabolic, jejunal phosphate and glucose transport affected by, weanling rat, 763

Aciduria, dicarboxylic, Zellweger syndrome and neonatal adrenoleukodystrophy, 62

Acyl-CoA dehydrogenase, variant medium chain, biosynthesis, cultured fibroblasts, 843

Adenosine triphosphatase, calcium-transporting enzyme, isolation in cystic fibrosis, 356

Adipose, metabolism, fetal and neonatal calf, 542

Adipose tissue, thymidine kinase activity, 118

Adolescence, food choices, Lipid Research Clinics prevalence study, 309

$\beta$-Adrenoceptor, function, leukocytes, neonate, 1152

Adrenoleukodystrophy, neonatal, dicarboxylic aciduria in, 62

Adult, erythrocyte filterability, compared with neonate, 1269

Aerosol, radiolabeled, submicronic, pulmo-

Age nary deposition sites, 1297

compensatory renal growth and, rat, 347

selenium status and, Belgium and The Netherlands, 574

Age differences

Cis-platin nephrotoxicity, rat, 9

plasma glutamate concentrations, 53

Aging, immunoreactive pancreatic cationic trypsinogen, changes related to, cystic fibrosis and pancreatic insufficiency, 209

Airway, collapsibility, effect of tracheal smooth muscle tone on, neonate, 492

Airway pressure, constant positive, assessment of optimal level, hyaline membrane disease, 884
Alanine, sodium absorption and, viral diarrhea, piglet, 879

Aldosterone, colonic NaKATPase activity, control, weanling rat, 242

American Pediatric Society

presidential address, 1345

medical ethics and the law, 913

Amino acid

branched-chain, metabolism, maple syrup urine disease, 169

concentration, maternal hypoaminoacidemia and, rat fetus, 1071

metabolism, insulin effect, fetal hepatocytes, rat, 609

pools, protein synthesis and, neonate, 140

sulfur uptake, membrane fluidity and brush border membrane, rat kidney, 1305

transport, brush border membrane, nursing animals, rat, 890

Amniotic fluid

digoxin and digitoxin-like immunoreactive substances, 28

isovalerylglycine in, stable isotope dilution analysis, prenatal isovaleric acidemia diagnosis, 222

Ampicillin, subinhibitory polymyxin B and, Haemophilus influenzae type B infection, infant rat, 237

Antibody, monoclonal, lymphocyte subsets identified by, healthy children, 1147

Apoprotein, plasma lipoprotein composition, neonates, 324

Arginine, insulin response, protein-energy malnutrition, 1122

Arginine vasopressin, urinary, excretion pattern, neonate, 103

Artery, cerebral, leukotriene effect, newborn pigs, 973

Arthritis, juvenile, complement activation patterns, 1332

Asphyxia

birth, neuroendocrine lung and brainstem injury, 828

effect on intestinal blood flow and oxygen uptake, newborn piglet, 929

neonatal, cerebral blood flow and $\mathrm{O}_{2}$ metabolism after, lamb, 778

perinatal, plasma $\beta$-endorphin in, 577

Astrocyte cell, synthesis and secretion of nerve growth-stimulating factor, neonatal mouse, 367

Audiometry, auditory brainstem response, treated congenitally hypothyroid children, 570

Benzoate, metabolic changes caused by, lysinuric protein intolerance, 1117

Bile, secretion, control, newborn puppy, 203

Bilirubin

conjugate formation, human newborn, 947

encephalopathy, role of total bilirubin, protein binding, and blood-brain barrier, rat, 789

unconjugated, auditory brainstem response, infant rhesus monkey, 511

Birth, cathecholamine release, effect of chemical sympathectomy, 1338
Birth weight, low, medium-chain triglycerides, organic acid excretion, 122

Blood, contamination, hemagglutination test, fetal red cells, 1082

Blood-brain barrier, role in bilirubin encephalopathy, rat, 789

Blood flow

as hematocrit function, neonatal lamb, 1274

cerebral

after asphyxia, newborn lamb, 778

alterations, prolonged hypocarbia, piglets, 147

autoregulation impairment, hypoxic newborn lamb, 516

sympathetic nerve stimulation effects on, newborn piglet, 131

intestinal, asphyxia, hypoxia, acidosis effect, newborn piglet, 929

pulmonary, tolazoline and, fetal lamb, 1131

regional, comparative effects, metabolic acidemia and hypoxemia, neonatal lamb, 756

regional cerebral, hyperventilation, newborn dog, 1102

Blood sampling, prenatal, normal fetus, 342

Body composition, determination, total body electrical conductivity method, neonate, 561

Body water, effect of extracorporeal membrane oxygenation on, neonatal baboon, 381

Bone

decreased mineral, chronic furosemide therapy, newborn rat, 74

growth and mineral content, very low birth weight infants, 925

Brain

blood flow, see Blood flow

cerebral cortical synaptosomes, nerve growth factor measurement, mouse, 391

cerebral metabolic intermediates, after intrauterine growth retardation, dog, 662

function, phenylalanine effects, phenylketonuria patients, 1112

hypothermic, nuclear magnetic resonance spectroscopy and, rabbit, 867

Brainstem

bombesin-like peptide regulation, pulmonary neuroendocrine cells, neonate, 828

methionine-enkephalin levels, hypoxia influence, rabbit, 655

Breast feeding

carnitine levels, serum and breast milk, 806

lymphocyte proliferation, effects on, infant, 227

see also Human milk

Breath amplitude, heart rate variability and, full-term neonates, 301

Breathing patterns, predictive value, sudden infant death syndrome, 680

Brush border membrane

amino acid transport, nursing animals, rat, 890

fluidity, glucocorticoid-mediated altera- 
tion, small intestine, rat, 79

Saccharomyces boulardii, response to, human and rat, 192

Cardiac output, comparative effects, metabolic acidemia and hypoxemia, newborn lamb, 756

Carnitine

phosphatidylcholine species composition and, fetal rat lung, 1280

serum and breast milk concentrations, during prolonged breast-feeding, 806

Carotid body, maturation, effect on laryngeal chemoreflex, neonatal lamb, 724

Catecholamine

hypoglycemia response, short stature and 1097

metabolic clearance and plasma appearance rates, fetal sheep, 992

release, chemical sympathectomy effect, 1338

Cell, endothelial, proliferation, mucocutaneous lymph node syndrome, 943

Cephalosporins, third generation, cellular uptake and cell-associated activity of, 909

Cerebral artery, Doppler ultrasound waveforms, controlled hypercapnia, neonate, 218

Cerebrospinal fluid, biogenic amine metabolites, acute lymphocytic leukemia, 285

Chemoreflex, laryngeal, effects of carotid body maturation and terbutaline, neonatal lamb, 724

Chemotaxis, neutrophil, ontogeny, fetal lamb, 265

Choking, first aid, comments, Howland Award acceptance, 1013

Cholecystokinin octapeptide, gallbladder contractility and, development, guinea pig, 214

Choline, uptake, intestine, neonatal and adult rat, 768

Circulation

nifedipine effects, newborn lamb, 1

pulmonary, prostaglandin $\mathrm{H}_{2}$ effects, perinatal lamb, 565

Cis-platin, nephrotoxicity, young and adult rats, 9

Coagulation, neonatal systems compared, lambs, piglets, rabbits, beagle pups, 961

Coagulopathy, acute polycythemia and, newborn dog, 151

Colon, NaKATPase activity, control, aldosterone role, weanling rat, 242

Complement

activation

different patterns, juvenile arthritis, 1332

respiratory fluids, cystic fibrosis, 1258

Concanavalin A, effect on peripheral blood mononuclear cells, glomerular basement membrane, rat, 321

Cord blood

digoxin- and digitoxin-like immunoreactive substances, neonate, 28

lipid levels, high-density lipoprotein subclass and, 487

mononuclear cells, interleukin-2 receptor production by, 136

Corticosteroid, globulin binding to, gestation, diabetic rat, 155

Corticosterone

circulating, thyroxine effects, infant rat, 87

fetal and maternal, corticosteroid binding globulin and, gestation, diabetic rat, 155

Creatinine clearance, effect of assisted ventilation, low birth weight infants, 447

Cromolyn sodium, pulmonary vasoconstriction and, neonatal lamb, 834

Cystic fibrosis

altered antibody isotope, opsonic deficiency and, 453

calcium-transporting ATPase enzyme isolation, erythrocyte membrane, 356

circulating immune complexes, correlation to clinical parameters, 385

diagnosis, premature infants, 536

linoleic acid deficiency, correction, 36

monocyte chemiluminescence in, patients and parents, 619

neutrophils, formyl-methionyl-leucylphenylalanine-induced response, 520

oxygen consumption and ouabain binding sites, nasal epithelium, 1316

pancreatic insufficiency and, immunoreactive pancreatic cationic trypsinogen, age-related changes, 209

phosphatidylethanolamine N-methyltransferase, properties and activity, 1290

Pseudomonas mucoid strains, antibody, enzyme-linked immunosorbent assay, 1085

respiratory fluids, complement activation in, 1258

Cystine, transport, developmental aspects, dog, 593

Dazmegrel, effects on pulmonary gas exchange, piglet, 481

Defense mechanism, pulmonary, postnatal maturation, lung, lamb, 496

Dehydroepiandrosterone sulfate, metabolism, growth retarded fetus, 166

Dexamethasone, superoxide dismutase turnover and, fetal lung, rat, 895

Diabetes

fetal and maternal corticosterone and corticosteroid binding globulin, gestation, rat, 155

streptozotocin-induced, lung development, fetal rat, 67

Diaphragm, force output, postnatal changes, piglet, 658

Diarrhea, sodium absorption, alanine effect, piglet, 879

Digitoxin, immunoreactive substances similar to, amniotic fluid, cord blood, and serum, neonate, 28

Digoxin, immunoreactive substances similar to, amniotic fluid, cord blood, and serum, neonate, 28

Dihydroxyvitamin $D_{3}$, see Vitamin $D$

Diltiazem, heart function and, neonate, 838

Diphtheria toxoid, IgG and IgG subclass specific antibody responses, DTP immunization, neonates and infants, 735

Disaccharidase, intestinal, nutritional rehabilitation effect, weanling rat, 793

Electrical conductivity, total body, body composition determination, neonate, 561

Electroencephalogram, phenylalanine effects, phenylketonuria patients, 1112

Electrolyte balance, effect of assisted ventilation, very low birth weight infants, 447

Embryo, environmental hyperthermia, hemodynamic effects, chick, 1213
Endocrine pulse, detection, dual-threshold method for, 632

$\beta$-Endorphin, plasma, perinatal asphyxia 577

Endotoxin, subinhibitory polymyxin B and ampicillin effects, Haemophilus influenzae type B infection, infant rat, 237

Energy

expenditure, estimation of, premature infants, 646

metabolism

in premature infants, 638

variations, preterm infants, 422

Enterotoxin

heat-stable, Escherichia coli, sensitivity, small intestine, immature rat, 555

inhibition by milk gangliosides, human and cow's milk compared, 416

Enzyme

antioxidant

lung development, streptozotocin-induced diabetes, fetal rat, 67

metyrapone effect on maturation, rat lung, 672

gluconeogenic, effects of fasting on, ovine fetus, 676

postreceptor defect, leprechaunism and, 329

Enzyme-linked immunosorbent assay, antibody detection, Pseudomonas mucoid strains, cystic fibrosis, 1085

Epidermal growth factor

artificial feeding and, suckling rat, 1248

differential response, neonatal rat, 468

lung development influenced by, fetal rat 473

neonatal hyperthyroidism effect

mouse saliva, 587

tissues, urine, and blood, mouse, 628

thyroxine effect, neonatal mice, 281

thyroxine-mediated responses, developing sympathetic nervous system involvement, submandibular gland, mouse, 232

Epinephrine, systemic blood pressure effect sepsis-induced pulmonary hypertension, piglet, 872

Epithelium, nasal, oxygen consumption and ouabain binding sites, cystic fibrosis 1316

Erratum, 160

Erythrocyte

cell flow during hypoxemia, fetal scalp, sheep, 407

deformability, fetus, preterm, and term neonate, 93

filterability, neonates and adults, 1269

insulin binding, preterm newborn infant, 256

neonatal, aging process, 1091

Erythropoiesis, infants of diabetic mothers, 161

Erythropoietin, immunoreactive, temporal response to acute hypoxemia, fetal sheep, 15

Escherichia coli, heat-stable enterotoxin sensitivity, small intestine, immature rat, 555

Escherichia coli $\mathrm{K} 1$, infection, protection against, neonatal rat, 175

European Society for Paediatric Gastroenterology and Nutrition, annual meeting abstracts, 689

European Society for Pediatric Research abstracts, 1034

author index to abstracts, 1065

lectures, 1017

Eye, fetal visual evoked potential, visual maturation and, lamb, 351 
Fasting, gluconeogenic enzymes and, ovine fetus, 676

Fatty acid

dicarboxylic and $\omega-1$ hydroxy, excretion, medium-chain triglyceride ingestion and, low birth weight infant, 122

hydroxylation, acetylsalicylic acid effect, rat liver, 1221

Feeding

artificial, epidermal growth factor and, suckling rat, 1248

thermogenic effect, premature infants, 638

Fertility, onset, undernutrition and, rat, 1161

Fetal alcohol syndrome, zinc status of infants with, 551

Fetus

acute hypoxemia, immunoreactive erythropoietin, temporal response, sheep, 15

adipose metabolism, calf, 542

amino acid concentrations, maternal hypoaminoacidemia and, rat, 1071

blood group antigens, maternal blood contamination detection, 1082

catecholamine clearance, sheep, 992

erythrocyte deformability, 93

gluconeogenesis and oxygenation, lamb, 532

gluconeogenic enzyme, fasting effect, sheep, 676

growth retardation

feto-placental steroid metabolism, 166

somatomedin $\mathrm{C} /$ insulin-like growth factor I concentrations, rat, 126

isolated hepatocytes, insulin effect, rat, 609

lung development

epidermal growth factor influence, rat, 473

nonhuman primate, male and female, 987

prolactin role, 876

streptozotocin-induced diabetes, rat, 67

thyroid hormone and, rat, 545

lung hypoplasia

morphometry, guinea pig, 955

oligohydramnios-induced, guinea pig, 951

lung surfactant and inositol, gestational age-dependent changes, rat, 24

metabolic requirements, guinea pig, 1156

mother and, corticosterone and corticosteroid binding globulin, gestation, diabetic rat, 155

neutrophil chemotaxis, ontogeny, lamb, 265

normal, prenatal blood sampling, 342

phosphatidylcholine species composition, thyroxine and carnitine effects, rat lung, 1280

prostaglandin concentrations, late gestation, sheep, 83

protein turnover, after maternal starvation, rat, 1252

pulmonary blood flow, tolazoline and, lamb, 1131

red blood cell flow, hypoxemia, scalp, sheep, 407

serum somatomedin $\mathrm{C}$, thymidine, and transferrin, 71

somatomedin-C/insulin-like growth factor I, tissue and plasma concentrations, first half of gestation, 253

superoxide dismutase turnover, dexamethasone effect, rat lung, 895

visual evoked potential, visual maturation and, lamb, 351

Fibrinogen, clottable, acute polycythemia effects, newborn dog, 151

Fibroblasts

branched-chain amino acids in, metabolism, maple syrup urine disease, 169

isovaleric acidemia, isovaleryl-CoA dehydrogenase activity, tritium release assay, 59

$\mathrm{N}$-acetyl-neuraminic acid distribution, infantile sialic acid storage disease, 773

Fluid, respiratory, complement activation in, cystic fibrosis, 1258

Folic acid, constant rate administration, neural tube defects and, 761

Food consumption, among white adolescents, Lipid Research Clinics prevalence study, 309

Formula

lipid differences, compared with breast milk, neonates, 332

triglycerides in, relation to plasma ketone body concentrations, 338

Freund's adjuvant, glucose transport following, rat kidney, 443

Furosemide, chronic therapy, growth failure and decreased bone mineral, newborn rat, 74

Gallbladder, contractility, development, guinea pig, 214

Ganglioside, composition compared, human and bovine milk, 416

Gestation

chemical composition, fetal and maternal guinea pig, 1156

fetal and maternal corticosterone and corticosteroid binding globulin, diabetic rat, 155

first half, somatomedin-C/insulin-like growth factor $\mathrm{l}$, tissue and plasma concentrations, fetus, 253

Gestational age

plasma inositol levels and surfactant composition, changes dependent on, fetal rat, 24

prostaglandin concentrations, maternal and fetal tissues, sheep, 83

protein synthesis and amino acid pools, neonate, 140

Gliadin, wheat, peptides, adenylate cyclase activation by, atrophic celiac intestinal mucosa, 53

Globulin, corticosteroid binding, fetal and maternal, gestation, diabetic rat, 155

Glucocorticoids

fluidity alteration mediated by, brush border membranes, small intestine, rat, 79

prostaglandins vs., small intestine, infant rat, 109

Gluconeogenesis

hepatic, oxygenation and, fetal lamb, 532

lactate related, small intestinal mucosa, suckling rats, 1321

Glucose

endogenous production, regulation, glucose role in, newborn, 49

hypertonic feeding, vascular response, mesentery, neonatal piglet, 1136

metabolism, perirenal adipose tissue, fetal and neonatal calf, 542

placental distribution, maternal concentration and, 269

polymer

absorption and oxidation, neonates, 740 hydrolysis, salivary amylase contribu- tion to, premature infant, 186

transport

alterations after Freund's adjuvant, kidney, rat, 443

metabolic acidosis effect, weanling rat, 763

uterine uptake, near-term guinea pig, 730

Glutamate, plasma concentrations, age differences, 53

Glycogenesis, type IB, chemotaxis and neutrophil function in, 438

Granulomatous disease, chronic, nitroblue tetrazolium test, eosinophils, 378

Growth and development

biosynthetic somatomedin $\mathrm{C}$ and, dwarf mouse, 825

chemical composition determination, fetal and maternal guinea pig, 1156

corticosterone, thyroxine effects, infant rat, 87

gallbladder contractility, guinea pig, 214

immune system development, premature infant, 899

lung, streptozotocin-induced diabetes, fetal rat, 67

myoinositol uptake, lung cells, perinatal rabbit, 179

sympathetic nervous system, thyroxinemediated nerve growth factor and epidermal growth factor responses, submandibular gland, mouse, 232

Growth factor

nerve

neonatal hyperthyroidism effect, mouse saliva, 587

submandibular gland, thyroxine effect, neonatal mouse, 281

$\beta$-nerve, measurement in cerebral cortical synaptosomes, mouse, 391

Growth failure, decreased bone mineral and, chronic furosemide therapy, newborn rat, 74

Growth hormone

arginine infusion and, protein-energy malnutrition, 1122

secretion

catch-up growth control and neural control, dissociation of, stunted head-irradiated rat, 261

catecholamines and, short children, 1097

Growth-promoting activity, serum somatomedin C, transferrin, and, fetus, 71

Growth retardation

feto-placental steroid metabolism, fetus, 166

intrauterine, cerebral metabolic intermediate response after, dog, 662

uterine artery ligation, somatomedin $\mathrm{C} /$ insulin-like growth factor I and, fetal rat, 126

Growth-stimulating factor, nerve, synthesis and secretion by astrocyte cells, neonatal mouse, 367

Guanylate cyclase, leprechaunism, postreceptor defect and, 329

Hearing

auditory brainstem response

treated congenitally hypothyroid children, 570

unconjugated bilirubin infusion, infant rhesus monkey, 511

Heart

mechanical function, $\mathrm{Ca}^{2+}$ channel blocker effect, neonate, 838

Purkinje fibers, autonomic interactions, 
dog, 613

rate, predictive value, sudden infant death syndrome, 680

verapamil, cardiovascular effects, age and, neonatal swine, 428

Heart rate, variability, respiration patterns and, normal neonate, 301

Heart ventricle

left, effects of interventricular shunt, dog, 433

septal defect, indices of left ventricular function, dog, 433

Hemagglutination, detection of maternal blood contamination, fetal red cells, 1082

Hematocrit, blood flow and oxygen delivery, neonatal lamb, 1274

Hematopoiesis, in vitro studies, pancytopenia in propionic acidemia, neonate, 783

Hemofiltration, end stage renal disease, vitamin D supplement effects, 5

Hemorrhage, intracranial, regional cerebral ischemia, potassium and, 478

Hepatocytes, amino acid and lipid metabolism in, insulin stimulation, fetal rat, 609

Herpes simplex

immune response, herpes labialis patients, 905

$\gamma$-interferon production and, neonatal mononuclear cells, 398

Howland Award

acceptance, first aid for choking victims,

presentation, 1986, Richard L. Day, 1009

Human milk

carnitine levels, prolonged breast feeding, 806

fortified, excretion of immune factors and, very low birth weight infants, 711

ganglioside composition, compared with cow's milk, 416

leukocytes, response to chemoattractant peptides, 373

lipid differences, compared with formula, neonates, 332

Hyaline membrane disease

constant positive airway pressure, assessment of optimal level, 884

recovery, surfactant quantity and composition during, 1243

Hydrolysis, glucose polymer, salivary amylase contribution to, premature in fant, 186

Hyperammonemia

congenital, behavioral and neurotransmitter changes, rat model, 1310

waste nitrogen excretion, benzoate and phenylacetate role, 1117

Hypercapnia, controlled, neonatal cerebral artery Doppler ultrasound waveforms, 218

Hyperimmunoglobulin $\mathrm{E}$ syndrome, intercurrent measles infection, transient remission after, 685

Hyperinsulinemia, lipase activities, infants of diabetic mothers, 527

Hypertension

pregnancy-induced, pituitary function, fetus, 876

pulmonary

pediatric cardiology workshop proceedings, 811

sepsis-induced, epinephrine effect, piglet, 872

Hyperthermia, environmental, hemody- namic effects, chick embryo, 1213

Hyperthyroidism

growth factor secretion and, saliva, neonatal mice, 587

neonatal, epidermal growth factor levels after, mouse, 628

Hyperventilation, cerebral blood flow during, newborn dog, 1102

Hypoaminoacidemia, maternal, amino acid concentrations and, fetal plasma, rat, 1071

Hypocarbia, prolonged, cerebral blood flow and oxygen consumption changes during, piglet, 147

Hypoglycemia, catecholamine response to, short stature and, 1097

Hypothermia, cerebral metabolic studies after, nuclear magnetic resonance spectroscopy and, rabbit, 867

Hypothyroidism, congenital, auditory brainstem response in, 570

Hypoxemia

effect on cardiac ouput and regional blood flow, unanesthetized newborn lamb, 756

immunoreactive erythropoietin, tempora response, fetal sheep, 15

neonatal status epilepticus and, nuclear magnetic resonance study, dog, 581

red blood cell flow, fetal scalp, sheep, 407

Hypoxia

alveolar, cromolyn sodium and, neonatal lamb, 834

cerebral blood flow autoregulation and, neonatal lamb, 516

chronic, in utero, pulmonary vasculature, neonatal guinea pig, 292

effect on intestinal blood flow and oxygen uptake, newborn piglet, 929

methionine-enkephalin levels and, brainstem nuclei, rabbit, 655

nonischemic, mucosal structure and function, dog jejunum, 1143

pulmonary vasoconstriction induced by, attenuation, newborn lamb, 113

$d$-tubocurarine and pancuronium, cardiovascular effects, newborn lamb, 246

vascular smooth muscle cell growth inhibited by, 966

ventilatory response, prenatal starvation and, neonatal guinea pig, 920

Immune complex

circulating, correlation to clinical parameters, cystic fibrosis, 385

immunoglobulin rheumatoid factor, in juvenile rheumatoid arthritis patients, 977

Immune factors, fecal excretion, fortified human milk and, very low birth weight infants, 711

Immune system, development, premature infants, 899

Immunoglobulin

Escherichia coli $\mathrm{K} 1$ infection, protection against, neonatal rat, 175

maternal intravenous infusion, transplacental passage, mothers to neonates, 933

plasma concentrations, immune system development, premature infants, 899

Immunoglobulin A, deficiency, with immunoglobulin G subclass deficiency, 937

Immunoglobulin $\mathrm{G}$

antibody responses, DTP immunization, neonates and infants, 735

subclass antibodies, in cystic fibrosis, 453 subclass deficiency, in immunoglobulin A deficiency, 937

Immunoreactivity

digoxin- and digitoxin-like substances, amniotic fluid, cord blood, and serum, neonate, 28

erythropoietin, temporal response to acute hypoxemia, fetal sheep, 15

pancreatic cationic trypsinogen, age-related changes, cystic fibrosis and pancreatic insufficiency, 209

Indomethacin, intestinal ulcer and, neonatal rat, 598

Infant

see also Neonate

macrosomic, lipase activities, 527

plasma peptide YY levels, 1225

Infantile sialic acid storage disease, $\mathrm{N}$-acetyl-neuraminic acid, distribution and metabolic fate, human fibroblasts, 773

Infection

patterns, immune system development, premature infants, 899

respiratory, immunoglobulin $\mathrm{G}$ subclass deficiency and, 937

see also specific infection

Inhalation, aerosol, radiolabeled, submicronic, pulmonary deposition sites, 1297

Inositol, plasma, gestational age-dependent changes, fetal rat, 24

Insulin

arginine infusion and, protein-energy malnutrition, 1122

binding, erythrocyte, preterm newborn infant, 256

islet-stimulating activity, obesity and, 720

Interferon, immune response to herpes simplex and, herpes labialis patients, 905

$\gamma$-Interferon, herpes simplex stimulation and, neonatal mononuclear cells, 398

Interleukin-2 receptor, see receptor

Intestinal mucosa

atrophic celiac, adenylate cyclase activation, wheat gliadin-derived peptides, 42

Saccharomyces boulardi, response to, human and rat, 192

Intestine

acute yersiniosis, clinical, morphological, and biochemical alterations, rabbit, 602

choline uptake by, neonatal and adult rat 768

repair, chronically undernourished rabbits, 1301

small

brush border membrane, glucocorticoid-mediated alteration of fluidity, rat, 79

Escherichia coli heat-stable enterotoxin and, rat, 555

glucocorticoid and prostaglandin effects, infant rat, 109

Irradiation, head, growth hormone secretion, rat, 261

Isovaleryl-CoA dehydrogenase, tritium release assay measuring isovaleric, acidemia fibroblasts, 59

Isovalerylglycine

stable isotope dilution analysis prenatal diagnosis of isovaleric acidemia diagnosis, 222

Jejunum, mucosal structure and function, 
nonischemic hypoxia effects, dog, 1143

Kawasaki disease, acute, endothelial cell proliferation in, 943

Ketoacid dehydrogenase, branched-chain, activity abnormalities, amino acid metabolism, 169

Kidney

amino acid transport, nursing animals, rat, 890

assisted ventilation and, very low birth weight infants, 447

compensatory growth, influence of age, rat, 347

cystine transport, developmental aspects, dog, 593

end stage disease, hemofiltration-treated, vitamin $D$ supplements and, 5

glucose transport, Freund's adjuvant and, rat, 443

hypercapnic acidosis and renal function, newborn rabbit, 798

$\beta$-Lactamase, inducibility, piperacillin susceptibility of Pseudomonas cepacia, cystic fibrosis, 1174

Lactate

gluconeogenesis and, small intestinal mucosa, suckling rats, 1321

metabolism, perirenal adipose tissue, fetal and neonatal calf, 542

uterine uptake, near-term guinea pig,, 730

Lactoferrin, isolation and characterization, monkey, 197

Lectures, European Society for Pediatric Research, 1017

Leprechaunism, guanylate cyclase activity, postreceptor defect and, 329

Leukemia, acute lymphocytic, biogenic amine metabolites, cerebrospinal fluid, 285

Leukocyte

$\beta$-adrenoceptor function, neonate, 1152

human milk, response to chemoattractant peptides, 373

polymorphonuclear

adherence and chemotaxis, healthy and stressed neonates, 297

leukotriene B4 biosynthesis, cord blood, infants, children, and adults, 402

Leukotriene B4, biosynthesis, umbilical cord blood, infants, children, and adults, 402

Leukotrienes

cerebral arteries affected by, newborn pig, 973

role in group B streptococcal sepsis, piglet, 1004

Linoleic acid, deficiency, correction, cystic fibrosis, 36

Lipase, hepatic, macrosomic infants, 527

Lipid

metabolism, insulin effect, fetal hepatocytes, rat, 609

plasma lipoprotein composition, neonates, 324

Lipid peroxidation, vitamin $\mathrm{E}$ effects, neonatal rabbit, 505

Lipoprotein

high-density, cord blood lipid levels and, 487

plasma, postheparin activity, hyperinsulinemic infants and large-for-date infants, 527

Lipoteichoic acid, group B streptococcal, toxicity, rabbit and mouse, 1168
Liver, fatty acid hydroxylation, acetylsali-

Lung cylic acid effect, rat, 1221

capacity, transfusion effects, thalassemia major, 20

circulation, effects of prostaglandin $\mathrm{H}_{2}$, perinatal lamb, 565

defense mechanisms, antimicrobial, postnatal maturation, conventional and germ-free lamb, 496

development

Clara cell-specific protein and, rat, 802

nonhuman primate fetus, male and female, 987

streptozotocin-induced diabetes, fetal rat, 67

thyroid hormone and, fetal rat, 545

effect of passive smoking, lamb, 853

functional residual capacity determination, $\mathrm{N}_{2}$ washout method, neonate, 668

hypoplasia

morphometry, after oligohydramnios, fetal guinea pig, 955

oligohydramnios-induced, fetal guinea pig, 951

influence of epidermal growth factor, fetal rat, 473

microvascular pressure, micropuncture measurement, rabbit, 1107

myoinositol uptake development, perinatal rabbit, 179

natural surfactant clearance, effects of dose and species, neonatal rabbit, 1139

phosphatidylcholine species composition, thyroxine and carnitine effects, fetal rat, 1280

pulmonary deposition sites, inhaled, radiolabeled, submicronic aerosol, 1297

pulmonary hypertension

epinephrine effect, piglet, 872

pediatric cardiology workshop proceedings, 811

pulmonary vasoconstriction, cromolyn sodium effect, neonatal lamb, 834

superoxide dismutase turnover, dexamethasone effect, fetal rat, 895

surfactant and antioxidant enzyme maturation, metyrapone effect, rat, 672

surfactant phospholipids, changes in, normal and diabetic pregnancies, rat, 650

vascular structure, hypoxia effects, neonatal guinea pig, 292

Luteinizing hormone, pulsatile, release, error estimate definition, 632

Lymphocyte

proliferation, breast feeding effects on, infant, 227

subset identification, monoclonal antibodies, healthy children, 1147

Macrosomia, neonatal, lipase activities in 623

Malnutrition

pancreatine exocrine function and, 1236

postnatal, disaccharidase response to nutritional rehabilitation, weanling rat, 793

protein-energy, insulin response to arginine infusion, 1122

Maple syrup urine disease, branched-chain amino acid metabolism, fibroblasts, 169

Maternity

fetus and corticosterone and corticosteroid bind ing globulin, gestation, diabetic rat, 155

prostaglandin concentrations, late gestation, sheep, 83

glucose concentration, placental glucose distribution and, 269

McCune-Albright syndrome, ovarian function in, clinical and laboratory results, 859

Measles, hyperimmunoglobulin E syndrome and, transient remission after, 685

Membrane

fluidity, sulfur amino acid uptake and, brush border membrane, rat kidney, 1305

oxygenation, extracorporeal, effect on body water content, neonatal baboon, 381

Mesentery, hypertonic feeding, microvascular response, neonatal piglet, 1136

Metabolism, cerebral intermediate re sponse, after intrauterine growth retardation, dog, 662

Methionine-enkephalin, age-dependent hypoxia influence, brainstem nuclei, rabbit, 655

Metyrapone, surfactant and antioxidant enzyme maturation and, rat lung, 672

Micropuncture, vascular pressure measured

Milk by, rabbit lung, 1107

cow's, ganglioside composition and enterotoxin-inhibitory activity, compared with human milk, 416

monkey, lactoferrin isolation and characterization, 197

Monoamine metabolites, acute lymphocytic leukemia, cerebrospinal fluid, 285

Monocyte, chemiluminescence in, cystic fibrosis patients and parents, 619

Mononuclear cell, $\gamma$-interferon production, herpes simplex virus stimulated, neonate, 398

Mononuclear cells, cord blood, interleukin2 receptor production by, 136

Monosodium L-glutamate, plasma glutamate concentrations, age differences, 53

Muco-cutaneous lymph node syndrome, endothelial proliferation in, 943

Mucosa, jejunal, hypoxia effects, dog, 1143

Muscle, smooth, tracheal mechanics and neonate, 1216

Myelin, tubular, formation, surfactant and phospholipid extract adsorption related to, cow, 97

Myocardium, dysfunction, experimental septic shock, 1240

Myoinositol, uptake, perinatal development, lung, rabbit, 179

NaKATPase, colonic activity, control, al dosterone role, weanling rat, 242

Naloxone, septic shock and, newborn piglet, 707

National Heart, Lung, and Blood Institute, pediatric cardiology workshop proceedings, pulmonary hypertension, 811

Neonate

acute polycythemia causing coagulopathy, dog, 151

adipose metabolism, calf, 542

adrenoleukodystrophy, dicarboxylic aciduria in, 62

airway collapsibility, effect of tracheal 
smooth muscle tone on, 492

asphyxia, cerebral blood flow and $\mathrm{O}_{2}$ metabolism after, lamb, 778

bile secretion, control, puppy, 203

bilirubin conjugate formation, 947

blood flow and oxygen delivery, as hematocrit function, lamb, 1274

body composition, mother's smoking and, 716

body composition determination, total body electrical conductivity method, 561

body water content, extracorporeal membrane oxygenation and, baboon, 381

breast feeding, lymphocyte proliferation, effects on, 227

cerebral artery Doppler ultrasound waveforms, controlled hypercapnia and, 218

cerebral blood flow impairment, hypoxic lamb, 516

chronic furosemide therapy, growth failure and decreased bone mineral, 74

circulating corticosterone, age and thyroxine effects, rat, 87

coagulation systems, comparative study, lambs, piglets, rabbits, beagle pups, 961

digoxin- and digitoxin-like immunoreactive substances, amniotic fluid, cord blood, and serum, 28

effects of chronic in utero hypoxia, pulmonary vasculature, guinea pig, 292

endogenous glucose production, regulation, glucose role in, 49

epidermal growth factor

differential response, rat, 468

compared with adult, 1269

erythrocytes

aging process, 1091

filterability, compared with adult, 1269

erythropoiesis, diabetic mothers, 161

Escherichia coli $\mathrm{K} 1$ infection, protection against, rat, 175

full-term

breath amplitude modulation of heart rate variability, 301

erythrocyte deformability, 93

functional residual capacity measurement, $\mathrm{N}_{2}$ washout method, 668

glucocorticoids and prostaglandins, comparative effects, small intestine, rat, 109

glucose polymer, absorption and oxidation, 740

Haemophilus influenzae type B infection, subinhibitory polymyxin B and ampicillin for, rat, 237

heart function, calcium antagonist effect, 838

hypercapnic acidosis, effect on renal function, rabbit, 798

hyperinsulinemic, plasma lipoprotein and hepatic lipase activities, 527

hyperthyroid, epidermal growth factor levels after, mouse, 628

hypertonic feeding, vascular response, mesentery, neonatal piglet, 1136

hyperventilation, regional cerebral blood flow during, dog, 1102

hypoxia-induced pulmonary vasoconstriction, attenuation, lamb, 113

intestinal ulcer, indomethacin and, rat, 598

large-for-date, plasma lipoprotein and hepatic lipase activities, 527

laryngeal chemoreflex, effects of carotid body maturation and terbutaline on lamb, 724

leukocytes, $\beta$-adrenoceptor function, 1152

lipid differences, breast versus formula 332

lipid peroxidation in, vitamin $\mathrm{E}$ effects, 505

lung and brainstem injury, neuroendocrine cells, 828

macrosomic

lipase activities, 527

lipase activities in, 623

mononuclear cells, $\gamma$-interferon production by, 398

natural surfactant clearance, rabbit lung, 1139

nerve growth-stimulating factor, synthesis and secretion by astrocyte cells, mouse, 367

nifedipine, circulatory effects, lamb, 1

normoxia and hypoxia, $d$-tubocurarine and pancuronium, cardiovascular effects, lamb, 246

pancytopenia and propionic acidemia, hematologic evaluation, 783

parenterally fed, surgery effects on nitrogen metabolism, 32

plasma lipoproteins, apolipoprotein and lipid compostion, 324

plasma peptide YY levels, 1225

polymorphonuclear leukocyte adherence and chemotaxis, comparison, healthy and stressed, 296

premature, see also Prematurity immunoglobulin, transplacental passage, 933

serum thyroglobulin levels, 1001

$\alpha$-thalassemia in, 1077

thyroglobulin cord serum levels, 996

variations in energy metabolism, 422

prenatal starvation, ventilatory response to hypoxia and, guinea pig, 920

preterm, variations in energy metabolism, 422

protein synthesis and amino acid pools, 140

pulmonary vasculature, hypoxia effects, guinea pig, 292

pulmonary vasoconstriction, cromolyn sodium and, lamb, 834

pyruvate carboxylase deficiency, cross-reacting material (negative), 274

respiratory difficulties, plasma $\beta$-endorphin in, 577

respiratory distress syndrome, cardiopulmonary function measurement, 316

sepsis, dazmegrel effects, piglet, 481

smooth muscle contraction, tracheal me chanics and, 1216

status epilepticus, effects of hypoxemia on, 581

stressed, polymorphonuclear leukocyte adherence and chemotaxis, 296

submandibular gland nerve growth factor synthesis, thyroxine-mediated mouse, 281

sympathetic nerve stimulation, effects on cerebral blood flow, piglet, 131

term, erythrocyte deformability, 93

unconjugated bilirubin infusion, auditory brainstem response, rhesus monkey, 511

urinary arginine vasopressin, excretion pattern, 103

verapamil, cardiovascular effects, pig, 428

very low birth weight

bone growth with low bone mineral content in, 925

effect of assisted ventilation in, 447 effects of human milk feeding, 711

whole blood filterability, compared with adult, 1269

Nephrology, pediatric, research grant applications, National Institutes of Health, 1067

Nephrotoxicity, Cis-platin, young and adult rats, 9

Nerve

growth factor, thyroxine-mediated responses, developing nervous system involvement, submandibular gland, mouse, 232

growth-stimulating factor, synthesis and secretion, neonatal mouse astrocyte cell, 367

Nervous system, sympathetic, developing, submandibular gland nerve growth factor and epidermal growth factor responses, mouse, 232

Neural tube defect, arsenate-induced, folic acid administration and, 761

Neuroblastoma, antigenic analysis, human, 915

Neuroendocrine cells, brainstem injury and neonatal lung, 828

Neurotransmitter, metabolism, acute lymphocytic leukemia, 285

Neutrophil

adhesion abnormality, surface membrane proteins and, 361

chemotaxis, ontogeny, fetal lamb, 265

cystic fibrosis, formyl-methionyl-leucylphenylalanine-induced response, 520

nitroblue tetrazolium staining, screening, chronic granulomatous disease, 378

peptide receptor activation in, tosylamino-phenylethyl-chloromethylketone effect, 848

type IB glycogenesis, 438

Newborn, see Neonate

Nifedipine, circulatory effects, newborn lamb, 1

Nitroblue tetrazolium, eosinophil staining screening, chronic granulomatous disease, 378

Nitrogen, metabolism, surgery effects, parenterally fed neonates, 32

Normoxia, $d$-tubocurarine and pancuronium, cardiovascular effects, newborn lamb, 246

Nuclear magnetic resonance, neonatal status epilepticus, effect of hypoxemia on, dog, 581

Nutrition

disaccharidase response to feeding patterns, weanling rat, 793

food choices among white adolescents, Lipid Research Clinics prevalence study, 309

medium-chain triglycerides, organic acid excretion and, low birth weight in fant, 122

parenteral, surgery effects on nitrogen metabolism, 32

$\mathrm{N}_{2}$ Washout method, functional residual capacity measurement, neonate and small children, 668

Obesity, serum insulinotropic activity, compared with nonobese infants and children, 720

Oligohydramnios

lung hypoplasia and

influence of timing and gestational du-

ration, fetal guinea pig, 951

morphometry, fetal guinea pig, 955 
Ouabain, binding sites, nasal epithelium, cystic fibrosis, 1316

Oxygen

arterial, saturation, transfusion effects, thalassemia major, 20

cerebral metabolic rate, hyperventilation, newborn dog, 1102

consumption

alterations, prolonged hypocarbia, piglet, 147

nasal epithelium, cystic fibrosis, 1316

delivery, as hematocrit function, neonatal lamb, 1274

high, lung development, streptozotocininduced diabetes, fetal rat, 67

metabolism, after asphyxia, newborn lamb, 778

uptake, asphyxia, hypoxia, acidosis effect, newborn piglet, 929

uterine uptake, near-term guinea pig, 730

Oxygenation, hepatic gluconeogenesis and, fetal lamb, 532

$\mathrm{PaCO}_{2}, \mathrm{pH}$ and, hypoxia-induced pulmonary vasoconstriction and, newborn lamb, 113

Pancreas

exocrine function, malnutrition and reserpine treatment effects, 1236

insufficiency, immunoreactive pancreatic cationic trypsinogen, age-related changes, cystic fibrosis, 209

Pancuronium, cardiovascular effects, normoxia and hypoxia, newborn lamb, 246

Pancytopenia, propionic acidemia and, hematologic evaluation, neonate, 783

Peptide

atrial natriuretic, plasma levels, healthy children and cardiac disease, 1328

chemoattractant, human milk leukocyte response, 373

chemotactic receptor activation, tosylaminophenylethyl-chloromethylketone effect, human neutrophils, 849

wheat gliadin-derived, adenylate cyclase activation by, atrophic celiac intestinal mucosa, 42

Peptide YY, plasma levels, neonates and infants, 1225

Perinate, myoinositol uptake development, lung, rabbit, 179

Peroxisome, phytol feeding and, mouse, 411

$\mathrm{pH}$, arterial, hypoxia-induced pulmonary vasoconstriction attenuated by, newborn lamb, 113

Phenylacetate, metabolic changes caused by, lysinuric protein intolerance, 1117

Phenylalanine

effects on brain function, phenylketonuria patients, 1112

neutrophil response, cystic fibrosis, 520

Phenylketonuria, brain function, phenylalanine effects, 1112

Phosphate, transport, metabolic acidosis effect, weanling rat, 763

Phosphatidylcholine

species composition, thyroxine and carnitine effects, fetal rat lung, 1280

surfactant clearance, neonatal rabbit lung, 1139

Phosphatidylethanolamine $\mathrm{N}$-methyltransferase

granulocyte, properties and activity, cystic fibrosis, 1290

lymphocyte, properties and activity, cystic fibrosis, 1290
Phosphatidylglycerol, synthesis, surfactant, perinatal rabbit, 179

Phosphatidylinositol

gestational age-dependent changes, fetal rat, 224

synthesis, surfactant, perinatal rabbit, 179

Phospholipid

composition, sulfur amino acid uptake and, brush border membrane, rat kidney, 1305

extracts, adsorption, tubular myelin formation and, cow, 97

lung effluent, surfactant substitution effect, respiratory distress syndrome, 1228

surfactant, changes, fetal lung, normal and diabetic pregnancies, rat, 650

Phytol, peroxisome proliferation and, mouse, 411

Placenta, glucose distribution, maternal glucose concentration and, 269

Plasma

ketone bodies, triglycerides and, infant formula, 338

lipoprotein, apolipoprotein and lipid composition, neonates, 324

Polycythemia, acute, coagulopathy and, newborn dog, 151

Polymyxin B, ampicillin and, Haemophilus influenzae type $\mathrm{B}$ infection, infant rat, 237

Potassium

regional cerebral ischemia, intracranial hemorrhage, 478

total body, mother's smoking and, neonate, 716

Preeclampsia, newborn prolactin in, relation to fetal adrenal activity, 876

Pregnancy

diabetic

erythropoiesis in infants, 161

fetal corticosterone, rat, 155

surfactant phospholipids, fetal rat lung, 650

immunoglobulin, intravenous infusion, transplacental passage, preterm neonate, 933

Prematurity

cystic fibrosis diagnosis, 536

energy expenditure, estimation, 646

energy metabolism, thermogenic effect of feeding and, 638

erythrocyte deformability, 93

erythrocyte insulin binding, newborn infant, 256

evolution of primitive reflexes, 1284

glucose polymer hydrolysis, salivary amylase contribution to, infant, 186

immune system development, plasma immunoglobulin concentrations and, 899

$\alpha$-/thalassemia in, 1077

Pressure, transdiaphragmatic, postnatal changes, piglet, 658

Primates, nonhuman, fetal lung development, male and female, 987

Prolactin, fetal lung maturation and, normotensive and hypertensive mothers, 876

Prostaglandin

concentrations, maternal and fetal tissues, late gestation, sheep, 83

glucocorticoids vs., small intestine, infant rat, 109

Prostaglandin $\mathrm{H}_{2}$, effects on pulmonary circulation, perinatal lamb, 565

Prostaglandin synthetase, inhibition, group
B streptococcal shock, rabbit, 864

Protein

Clara cell-specific, quantitative assay, rat lung, 802

hydrophobic, surfactant activity, 460

pulmonary surfactant-associated, immunologic identification, 744

surface membrane, neutrophil adhesion abnormality and, 361

synthesis, amino acid pools and, neonate, 140

turnover, after maternal starvation, fetal rat tissue, 1252

Pseudomonas aeruginosa, mucoid strains, antibody, cystic fibrosis patients, 1085

Pseudomonas cepacia, piperacillin susceptibility, $\beta$-lactamase inducibility and, cystic fibrosis, 1174

Puberty

precocious, ovarian function, 859

undernutrition and, rat, 1161

Purkinje fibers, autonomic interactions, $\operatorname{dog}, 613$

Pyruvate carboxylase, deficiency, cross-reacting material (negative), infant, 274

Rebreathing, cardiopulmonary function measurement, respiratory distress syndrome, ventilated neonates, 316

Receptor

insulin, erythrocyte, premature newborn infant, 256

interleukin-2, cord blood mononuclear cells, 136

Red blood cell, see Erythrocyte

Reflex, primitive, evolution, premature in fant, 1284

Research, pediatric nephrology, grant applications, National Institutes of Health, 1067

Reserpine, chronic treatment, pancreatine exocrine function and, 1236

Respiration

dynamics, rapid rate mechanical ventilation, paralyzed rabbit, 750

heart rate variability and, normal fullterm neonates, 301

Respiratory distress syndrome

cardiopulmonary function measured, ventilated neonates, 316

lung effluent phospholipids, surfactant substitution effect, 1228

Rheumatoid factor, immune complexes, in juvenile rheumatoid arthritis patients, 977

Richard L. Day

Howland award acceptance, 1013

Howland award presentation, 1009

Saccharomyces boulardi, small bowel response to, human and rat, 192

Salivary amylase, glucose polymer hydrolysis, contribution to, premature infant, 186

Selenium, status as function of age, Belgium and The Netherlands, 574

Sepsis

dazmegrel effects, piglet, 481

group B streptococcal, leukotriene role, piglet, 1004

Septic shock, naloxone effect, newborn piglet, 707

Serum

digoxin and digitoxin-like immunoreac tive substances, neonate, 28 
Escherichia coli $\mathrm{K} 1$ infection, protection against, neonatal rat, 175

insulinotropic activity, obese and nonobese infants and children, 720

lipids, breast versus formula, 332

Shock

group B streptococcal, prostaglandin syn thetase inhibition in, rabbit, 864

septic, experimental, myocardial dysfunction comparison, 1240

Short stature, catecholamine response to hypoglycemia, 1097

Shunt, interventricular, effects on indices of left ventricular function, dog, 433

Smoking

maternal, total body potassium and, neonate, 716

passive, effect on lung, lamb, 853

Smooth muscle cell, vascular, hypoxia effect on growth, 966

Society for Pediatric Research, presidential address, 1348

Sodium, absorption, alanine and, piglet jejunum, 879

Somatomedin C

biosynthetic, growth effects, dwarf mouse, 825

serum, thymidine, transferrin, and, fetus, 71

Somatomedin-C/insulin-like growth factor I

tissue and plasma concentrations, first half of gestation, fetus, 253

tissue and serum concentrations, growth retardation and, fetal rat, 126

Spectroscopy, nuclear magnetic resonance, cerebral metabolic studies by, rabbit, 867

Spermatogenesis, undernutrition and, rat, 1161

Staining, nitroblue tetrazolium, screening, chronic granulomatous disease, 378

Starvation

maternal, protein turnover after, fetal rat tissue, 1252

prenatal, ventilatory response to hypoxia and, neonatal guinea pig, 920

Status epilepticus, neonatal, effect of hypoxemia on, dog, 581

Steroids, feto-placental metabolism, growth retarded fetus, 166

Streptococcus

group B

colonization prevention, rabbit and mouse, 1168

sepsis, hemodynamics, piglet, 1004

Submandibular gland, nerve growth factor, thyroxine-mediated, developing sympathetic nervous system involvement, mouse, 232

Sudden infant death syndrome, heart rate and breathing patterns, predictive value, 680

Sulfate, effect of concanavalin A on uptake, glomerular basement membrane, rat,

Superoxide dismutase, dexamethasone and, fetal lung, rat, 895

Surfactant

activity, hydrophobic protein and, 460

adsorption, tubular myelin formation, cow, 97

clearance rate, neonatal rabbit lung, 1139 composition

during hyaline membrane disease recovery, 1243

gestational age-dependent changes, fe- tal rat, 24

maturation, metyrapone effect on, rat lung, 672

phosphatidylglycerol and phosphatidylinositol synthesis, perinatal rabbit, 179

protein, hydrophobic, immunologic identification, 744

substitution, phospholipid lung effluent and, respiratory distress syndrome, 1228

Surgery, nitrogen metabolism, effects on, parenterally fed neonates, 32

Sympathetic nervous system

see also Nervous system, sympathetic

stimulation

effect on cerebral blood flow, newborn piglet, 131

Synaptosome, cerebral cortical, nerve growth factor measurement, mouse, 391

Terbutaline, effect on laryngeal chemoreflex, neonatal lamb, 724

Testicle, postnatal activity, salivary testosterone measurement and, 1324

Testosterone, salivary measurement, postnatal testicular activity and, 1324

Tetanus toxoid, IgG and IgG subclass specific antibody responses, DTP immunization, neonates and infants, 735

$\alpha$-Thalassemia, premature neonate, 1077

Thalassemia major, transfusion, pulmonary effects, 20

Thymidine, serum somatomedin C, transferrin, and, fetus, 71

Thymidine kinase, adipose tissue, 118

Thyroglobulin

cord serum levels, preterm infants, 996

serum levels, preterm infants, 1001

Thyroid hormone, fetal lung maturation, rat, 545

Thyrotropin-releasing hormone, pituitarythyroid axis response, mother, fetus, neonate, 982

Thyroxine

corticosterone development and, infant rat, 87

effect on submandibular gland nerve growth factor and epidermal growth factor, neonatal mice, 281

nerve growth factor and epidermal growth factor responses mediated by, developing sympathetic nervous system involvement, submandibular gland, mouse, 232

phosphatidylcholine species composition and, fetal rat lung, 1280

Tidal volume, measurement, high-frequency jet ventilation, rabbit, 45

Tolazoline, local infusion, pulmonary blood flow adaptation, fetal lamb, 1131

Tosylaminophenylethyl-chloromethylketone, chemotactic peptide receptor activation and, human neutrophils, 848

Trachea

smooth muscle, role of contraction, neonate, 1216

smooth muscle tone, effect on airway collapsibility, neonate, 492

Transferrin, serum somatomedin $\mathrm{C}$, thymidine, and, fetus, 71

Transfusion, pulmonary effects, thalassemia major, 20
Triglycerides

medium-chain

organic acid excretion and, low birth weight infant, 122

plasma ketone bodies and, infant formula, 338

Trypsinogen, immunoreactive pancreatic cationic, age-related changes, cystic fibrosis and pancreatic insufficiency, 209

$d$-Tubocurarine, cardiovascular effects, normoxia and hypoxia, newborn lamb, 246

Ulcer, intestinal, indomethacin and, neonatal rat, 598

Ultrasonography, Doppler, cerebral artery waveforms, controlled hypercapnia and, neonate, 218

Umbilical cord, polymorphonuclear leukocytes, leukotriene $\mathrm{B} 4$ biosynthesis in, infants, children, and adults, 402

Undernutrition

chronic, intestinal repair, rabbit, 1301

fertility onset, spermatogenesis, and pubertal development related to, rat, 1161

Urease, behavioral and neurotransmitter changes caused by, congenital hyperammonemia, rat model, 1310

Urine

arginine vasopressin, excretion pattern, neonate, 103

isovalerylglycine in, stable isotope dilution analysis, prenatal isovaleric acidemia diagnosis, 222

Uterine artery, ligation, growth retardation, somatomedin $\mathrm{C}$ /insulin-like growth factor I and, fetal rat, 126

Uterus, oxygen, glucose, and lactate movement, near-term guinea pig, 730

Vasoconstriction

pulmonary

cromolyn sodium and, neonatal lamb, 834

hypoxia-induced, attenuation, newborn lamb, 113

Ventilation

assisted, renal function and, premature infants, 447

high-frequency jet, tidal volume measurement during, rabbit, 45

mechanical, rapid rate, respiration $\mathrm{dy}$ namics during, paralyzed rabbit, 750 newborn guinea pig, 920

Verapamil

age-dependent cardiovascular effects, neonatal swine, 428

heart function and, neonate, 838

Vitamin $\mathrm{D}, 1,25$-dihydroxyvitamin $\mathrm{D}_{3}$, mineral balance and, hemofiltrationtreated end stage renal disease, 5

Vitamin $\mathrm{E}$, lipid peroxidation and, neonatal rabbit, 505

Whole blood, filterability, neonates and adults, 1269

Yersiniosis, intestinal response, rabbit, 602

Zellweger syndrome, neonatal adrenoleukodystrophy and, dicarboxylic aciduria in, 62

Zinc, fetal alcohol syndrome infants, 551 